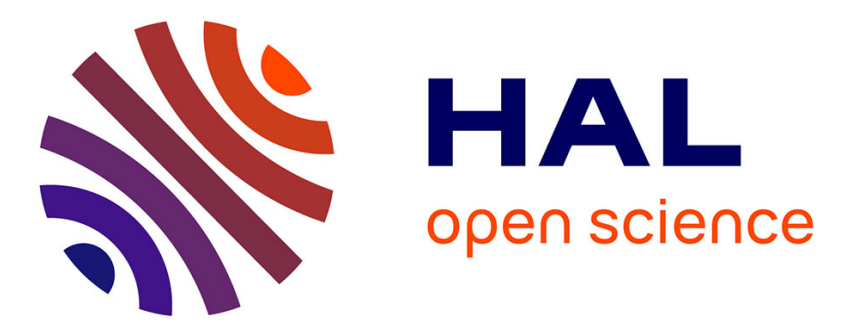

\title{
Rotor-stator interaction scenarios for the centrifugal compressor of a helicopter engine
}

Alain Batailly, Markus B. Meingast, Mathias Legrand, Jean-Philippe Ousty

\section{To cite this version:}

Alain Batailly, Markus B. Meingast, Mathias Legrand, Jean-Philippe Ousty. Rotor-stator interaction scenarios for the centrifugal compressor of a helicopter engine. ASME IDETC Conference, Aug 2013, Portland, United States. pp.IDETC-12211, 10.1115/DETC2013-12211 . hal-00851396

\section{HAL Id: hal-00851396 https://hal.science/hal-00851396}

Submitted on 20 Aug 2013

HAL is a multi-disciplinary open access archive for the deposit and dissemination of scientific research documents, whether they are published or not. The documents may come from teaching and research institutions in France or abroad, or from public or private research centers.
L'archive ouverte pluridisciplinaire HAL, est destinée au dépôt et à la diffusion de documents scientifiques de niveau recherche, publiés ou non, émanant des établissements d'enseignement et de recherche français ou étrangers, des laboratoires publics ou privés.

\section{(c)(1)}

Distributed under a Creative Commons Attribution| 4.0 International License 


\title{
Scénarios d'interaction rotor/stator pour un compresseur haute pression d'un moteur d'hélicoptère
}

\begin{abstract}
A. Batailly, Laboratoire de dynamique des structures et vibrations, université McGill, Québec M. Meingast, Laboratoire de dynamique des structures et vibrations, université McGill, Québec M. Legrand, Laboratoire de dynamique des structures et vibrations, université McGill, Québec J.-P. Ousty, Turbomeca, France
\end{abstract}

\section{Résumé}

Cette étude porte sur les phénomènes d'interaction pouvant apparaître au niveau du compresseur centrifuge de moteurs d'hélicoptères lorsque des contacts se produisent entre les sommets d'aubes et le carter environnant. De tels phénomènes peuvent engendrer de hauts niveaux de vibrations et sont présentement étudiés pour les compresseurs axiaux de moteurs d'avions pour lesquels des ruptures d'aubes ont été observées expérimentalement. Les avancées récentes ont permis d'améliorer la compréhension des phénomènes et ainsi d'envisager des simulations numériques sur des géomètries plus complexes telles que celles des compresseurs centrifuges. La simulation de contacts aube/carter est effectuée à partir d'une stratégie déjà présentée dans la littérature. Un carter ovalisé est utilisé et deux configurations de contact sont étudiées: (1) seule une aube peut entrer en contact avec ce carter et (2) toutes les aubes peuvent entrer en contact avec le carter. Pour chaque scénario, les résultats obtenus sont analysés dans les espaces temporel et fréquentiel. La plausibilité d'un scénario d'interaction n'impliquant qu'une seule aube, sur le modèle de ce qui est observé pour les compresseurs axiaux, est mise en évidence. Lorsque le contact est pris en compte entre le carter et l'ensemble des aubes du compresseur, les interactions observées sont plus complexes et les critères linéaires habituellement considérés ne permettent plus de prévoir les zones d'interaction.

\section{Rotor-stator interaction scenarios for the centrifugal compressor of a helicopter engine}

\begin{abstract}
The present paper deals with interaction phenomena that may arise in the centrifugal compressor of helicopter engines when structural contacts occur between the blade-tips and the surrounding casing. Those phenomena may lead to increased levels of vibration and are currently under investigation for axial compressors of aircraft engines for which blade failures were observed. The growing understanding of these phenomena allows for more challenging numerical simulations with increased geometrical complexity such as centrifugal compressors. The simulation of blade-tip/casing contacts is carried out based on an existing strategy previously introduced by the authors. An ovalized casing is considered and two contact configurations are investigated: (1) a single blade impacts the casing and (2) contact may occur on any blade of the cyclic assembly. Based on these two contact scenarios, an in-depth analysis is performed through both time and space Fourier transforms. Results highlight the plausibility of blade/casing interaction phenomena similar to those observed in axial compressors since interactions are detected with localized vibration levels on a single impacting blade. When contact is treated on all the blades of the centrifugal compressor, the witnessed interactions feature a higher degree of complexity and usual criteria become insufficient to predict interaction speeds as well as the location of significant peaks of vibration in the frequency domain.
\end{abstract}




\section{Introduction}

Aircraft or helicopter engines feature relatively small operating clearances that, under extreme operating conditions, may be consumed. Consequently, contacts can occur between the rotating components - such as bladed disks and impellers - and their surrounding casings. Depending on the rotational frequency of the rotating component, some interaction phenomena may arise such as the ones reported in [1] in the case of aircraft engines. Because they may lead to blade failure [1] and increased vibratory levels which may damage the structure, a significant amount of work has been dedicated to the understanding of these phenomena as well as the development of strategies for avoiding them. The repetability of such experiments as well as the possibility to obtain accurate results from numerical simulations [2] indicate that the interaction phenomenon is possibly robust. It is thus of great interest to assess the severity of this phenomenon for other types of compressor such as centrifugal compressors, also named impellers. Several references, both dealing with numerical simulations $[3,4,2]$ and experimental set-ups $[5,6,7]$, may be found on interaction phenomena or rubbing and abradable sealing properties in fan or axial compressors. However, the exploration of interaction phenomena for centrifugal compressors is not yet documented. The only references available deal with numerical $[8,9]$ and experimental $[10]$ modal analysis.

Contrary to axial compressors, centrifugal compressors feature a complex blade-tip profile which curvature calls for an even more precise unilateral contact procedure. In addition, contrary to axial compressors, impellers designs specificities involve a significant change of the blade height along the chord and the presence of disk modes even among very low frequency free-vibration modes. Because of these significant differences, the relevance of the interaction phenomenon reported in [1] for axial compressors may be questioned when adapted to centrifugal compressors. In particular, disk dynamics was experimentally proved to be negligible during the interaction with bladed disks (in aircraft axial compressors); such an assumption may not be pertinent for centrifugal compressors.

Accordingly, this study aims at evaluating the sensitivity of a given impeller geometry to potential interaction phenomena over its rotational frequency range. As mentioned previously, particular attention is paid to the interaction phenomena previously observed in axial compressors [1] that essentially involves a single blade. In this context, the present work focuses on the comparison of contact simulations between an impeller and a rigid casing with two contact scenarios: (1) contact only occurs on one main blade, in order to simulate the type of interaction described in [1] and (2) contact may occur on all the main blades of the impeller. As a first attempt to investigate such type of interaction phenomenon in a centrifugal compressor, the abradable liner is neglected.

The first section of the paper describes the interaction phenomenon of interest. Then the modeling of the impeller as well as the contact configuration are detailed in the second and third sections. Sections four and five focus on the analysis of the results obtained from the numerical simulations of blade/casing contact scenarios respectively involving a single blade and all the blades of the impeller.

Note: for the sake of confidentiality, all the frequencies and displacements given in this paper are normalized.

\section{Interaction phenomenon}

Blade rub has been observed in low-pressure (LP) and high-pressure (HP) axial compressors of a full scale turbojet [1]. The experiments were made under vacuum so that no aerodynamic load is applied on the blades. The experimental protocol and observations may be summed up as follows:

1. a slightly longer blade is instrumented in order to monitor the stress level; 
2. the rotor undergoes a sudden acceleration so that it reaches its nominal rotation speed which remains constant during the experiment;

3. blade/casing contact is initiated through the centrifugal load applied on the blade;

4. after a few seconds, significant vibration levels are monitored in the blade;

5. after about a minute, the stress level brutally increases until blade failure.

Measurements carried out after the experiment exhibit a complex wear pattern on the abradable liner deposited on the casing. For each experiment, a specific number of lobes over the circumference of the liner is obtained. The number of lobes varies depending on the type of blade considered. A numerical investigation of such occurrences has been conducted in [2] where the fundamental role of blade dynamics was underlined. Also, the need for a synchronization of the rotational frequency of the blade with its own frequency of vibration was highlighted.

Both experimental and numerical observations corroborate a negligible level of vibration of the casing - which is a consequence of a very low blade/casing rigidity ratio - as well as the bladed disk. Numerical simulations [2] could thus be carried out with a rigid casing and a blade clamped on its foot.

However, when dealing with specific rotor geometries such as centrifugal impellers, blade dynamics is intimately related to the disk dynamics which must be accounted for. Accordingly, the question may be raised of the plausibility of such interaction phenomenon - involving a single blade - for impellers.

\section{Modeling}
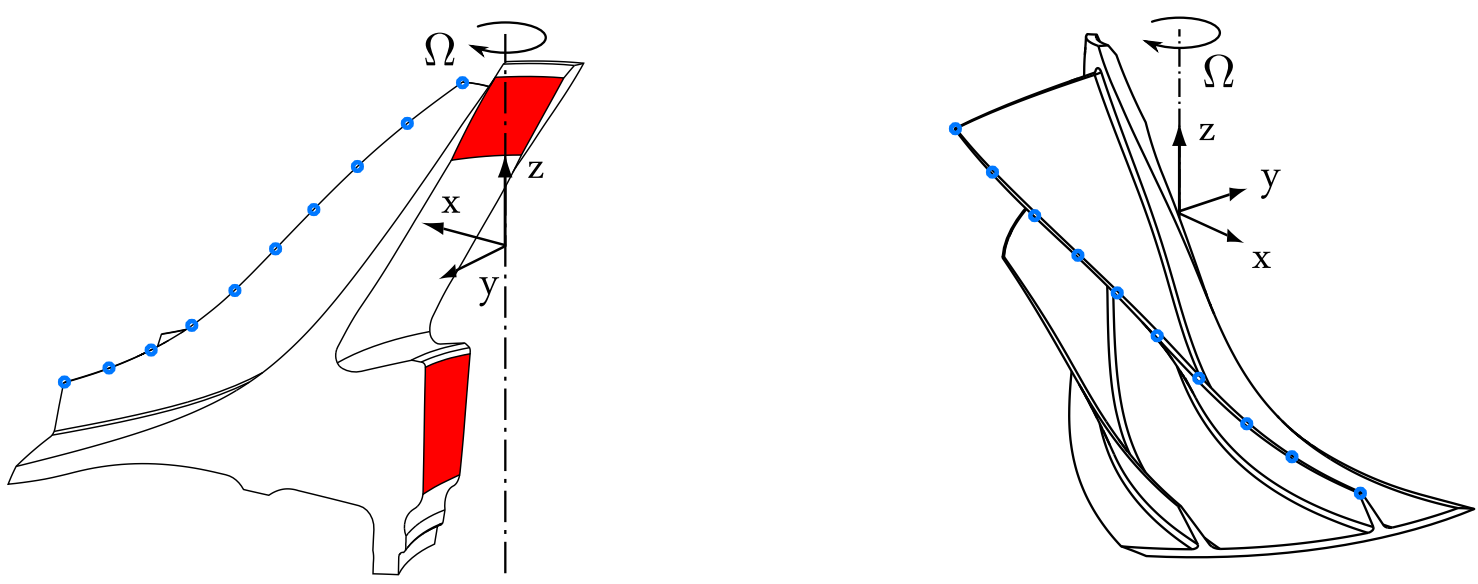

Figure 1 - ELEMENTARY CYCLIC SECTOR OF THE IMPELLER AND BOUNDARY CONDITIONS

Contact management relies on an explicit time integration scheme and a Lagrange multiplier based procedure [11] that was previously described in [12] and is not recalled here for the sake of brevity. The investigated impeller is assumed perfectly tuned and is composed of ten sectors. Each sector possesses a main blade and a splitter blade as pictured in Fig. 1. The finite element model of an elementary cyclic sector contains about 120,000 degrees of freedom (DOF).

Based on the cyclic symmetry of the impeller, its displacement field may be projected without any loss of information [13] over six spatial harmonics or nodal diameters $n_{d}$. The maximum number of nodal diameters of a cyclic symmetric structure is given by $n_{d, \max }=$ $\mathrm{E}\left(\frac{\mathrm{N}}{2}\right)+1$ where $\mathrm{E}(x)$ is the integer part of $x$ and $\mathrm{N}$ the number of sectors. In our study, $\mathrm{N}=10$ thus there is a total number of $n_{d, \max }=6$ distinct nodal diameters $n_{d}=0, \ldots, 5$. 
The free vibration modes of a cyclic symmetric structure are clustered in modal families [13]. Each modal family refers to a specific type of vibration of the blades (bending, torsion...) and contains 10 modes: the $i^{\text {th }}$ modal family contains the $i^{\text {th }}$ mode with 0 and 5 nodal diameters and the $(2 i)^{\text {th }}$ and $(2 i+1)^{\text {th }}$ modes with 1 to 4 nodal diameters. A free vibration mode of a cyclic symmetric structure is fully defined by the pair $\left(m, n_{d}\right)$ where $m$ is the number of the modal family it belongs to, and $n_{d}$ is its number of nodal diameters.

The dimension of the model does not allow for contact simulations within reasonable computation times. Consequently, component mode synthesis methods are used in order to reduce the size of the model: the Craig-Bampton [14] method is implemented in such a way that centrifugal stiffening is accounted for directly within the reduced-order model [15]. The reduced mass $\mathbf{M}_{r}$ and stiffness $\mathbf{K}_{r}(\Omega)$ matrices refer to a reduced model containing 900 DOFs:

$$
\begin{aligned}
& \mathbf{M}_{r}=\sum_{i}^{\mathrm{N}}\left(\mathbf{M}_{r}^{\left(n_{d}\right)}\right) \\
& \mathbf{K}_{r}(\Omega)=\sum_{i}^{\mathrm{N}}\left(\mathbf{K}_{0, r}^{\left(n_{d}\right)}+\Omega^{2} \mathbf{K}_{1, r}^{\left(n_{d}\right)}+\Omega^{4} \mathbf{K}_{2, r}^{\left(n_{d}\right)}\right)
\end{aligned}
$$

Contact is managed on ten boundary nodes, depicted in blue in Fig. 1, evenly spaced along the main blade-tip. A total of thirty DOF are used for contact treatment. These DOF are physically accessible in the reduced-order model thus avoiding costful mappings between the finite element space and the reduced space for the correction of the displacements when contact is detected.

Finally, boundary conditions are defined in such a way that all the nodes on the shaft/impeller interface are clamped as depicted in red in Fig. 1.

\section{Contact configurations}

The casing surrounding an impeller or any other type of compressor always features imperfections that do not make it perfectly circular. In addition, a casing undergoing thermal gradient - due to the temperature of the circulating gas - or maneuver loads tends to be ovalized. For that reason, the contact configuration considered in our study features a casing deformed along a two-nodal diameter mode with two main contact areas as depicted in Fig. 2.

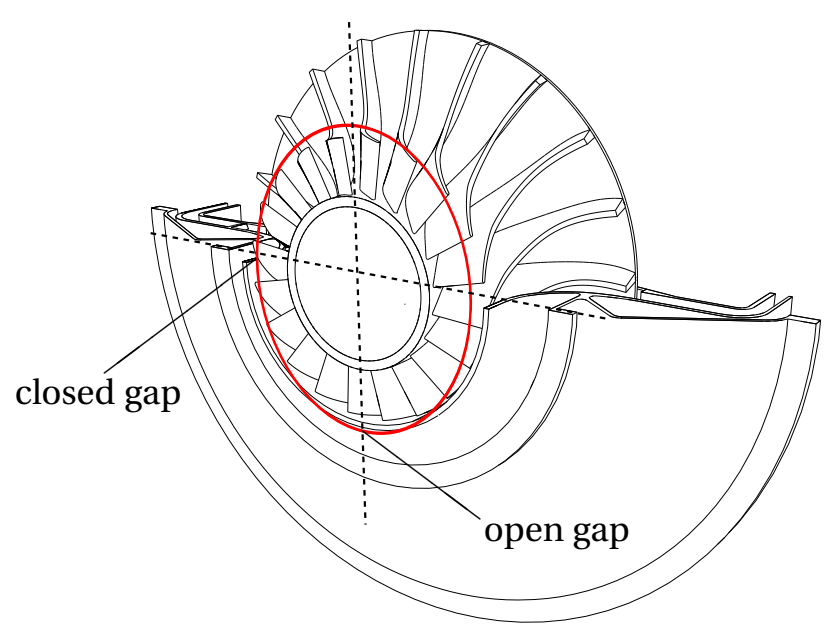

Figure 2 - SCHEMATIC REPRESENTATION OF THE OVALIZED CASING (-).

Accounting for the impefections of the casing goes beyond the scope of this study but the proposed methodology could account for them. 


\section{Single blade simulations}

The dynamics of the full impeller is accounted for but contact is managed on a single main blade only. Contrary to the numerical simulations in [2], the disk dynamics is accounted for. Contact simulations are carried out over the rotational frequency range of the impeller $\Omega \in[3.2 ; 5.95]$. For each rotational frequency, the impeller undergoes fifty revolutions within the ovalized casing. A Fourier transform of the radial displacement of the leading edge is computed from the steady-state response.

\subsection{Frequency domain}

Put side by side, the Fourier transforms obtained for each rotational frequency sketch the interaction map in Fig. 3. The black dashed lines represent the engine order (EO) lines, for which $f=k \Omega, k=1, \ldots, 11$. The rotation of the impeller in the ovalized casing induces a synchronization of the impeller vibratory response with the double of the rotational frequency that is the reason why even EOs are the location of the highest amplitude peaks in Fig. 3. Accordingly, even EOs may be seen as the main harmonics of the time signal in the frequency domain for each rotational frequency.

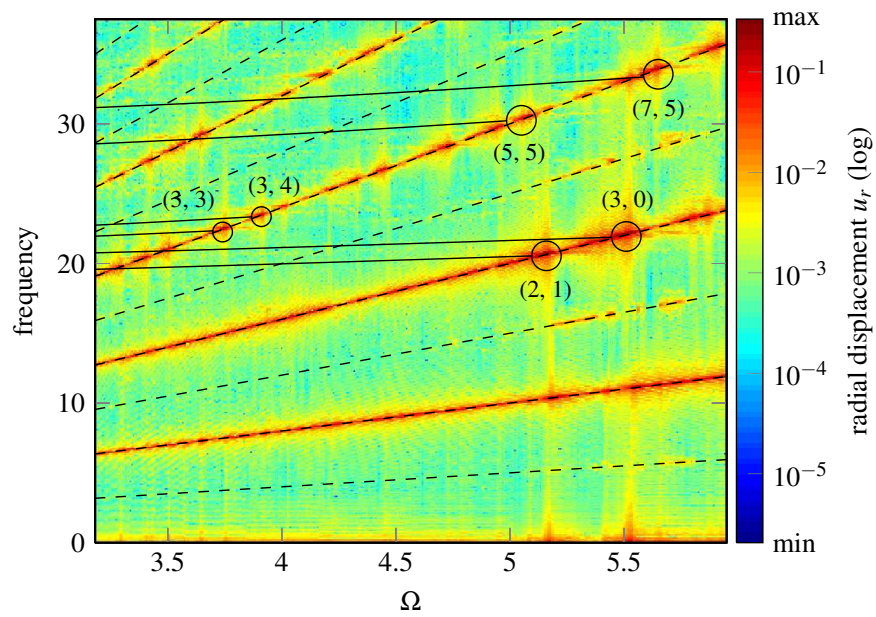

Figure 3 - INTERACTION MAP FOR 2 LOBES (SINGLE BLADE CONFIGURATION).

The eigenfrequencies of the structure are dependent on its rotational frequency due to centrifugal stiffening. The evolution of the eigenfrequencies is pictured as solid black eigenfrequency lines. Some of these lines are partially pictured in Fig. 3 for a few pairs $\left(m, n_{d}\right)$. In the interaction map, depending on the contact configuration, the intersection between the eigenfrequency lines and the EOs is a typical location for high amplitude peaks of vibration [12] which allow for the detection of critical rotational frequencies.

Several critical rotational frequencies are visible in Fig. 3 and marked with black circles, particularly around $\Omega=3.75, \Omega=5.15$, and $\Omega=5.5$.

\subsection{Harmonic tracking}

The impeller is a cyclic symmetric structure and its displacement field in the physical coordinates system may be transformed in the cyclic coordinates system.

\section{Principle}

For a given rotational frequency, a 2D Fourier transform of the time results may be carried out in order to highlight participating spatial and time harmonics as pictured in Figs. 4(a) and 4 (b). The name harmonic tracking is used in this paper to underline the relationship between the EOs (time harmonics) and the nodal diameters $n_{d}$ (spatial harmonics) through out the rotational frequency range. More precisely, this section focuses on: 


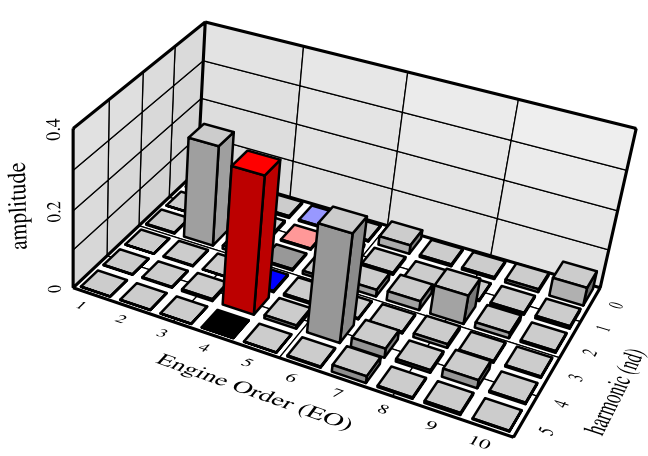

(a) $\Omega=5.2$ : nodal diameters tracking

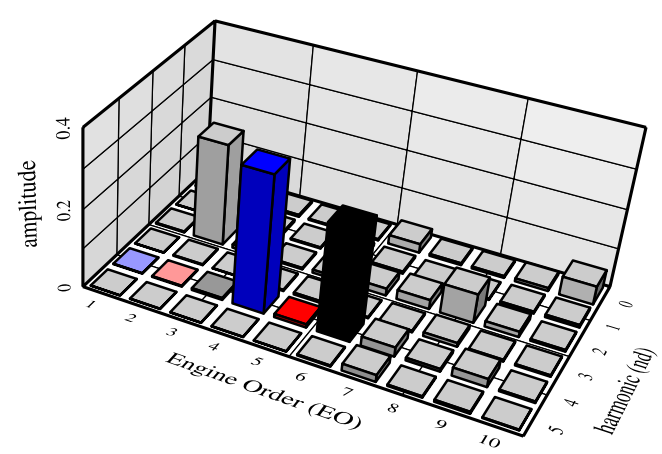

(b) $\Omega=5.2$ : EO tracking

Figure 4-2D Fourier transform of the time results.

1. tracking the influence of spatial harmonics (nodal diameters) for a given time harmonic (EO) (the evolution of the amplitude level of each of the six bars for a given EO in Fig. 4(a));

2. tracking the repartition of each spatial harmonics (nodal diameters) over the time harmonics (EO) (the evolution of the amplitude level of the first six bars for a given $n_{d}$ in Fig. 4(b)).

\section{Results}

For the sake of clarity, only EOs 1 to 6 are considered in the following. The colour code is similar to the one already introduced in Figs. 4(a) and 4(b). It is clear in Fig. 3 that higher EOs contain peaks of smaller amplitude thus not accounting for them does not significantly alter the accuracy of the results. The influence of a spatial (respectively time) harmonic at a given rotational frequency and for a given time (respectively spatial) harmonic is defined by the ratio (in \%) of the response amplitude for this harmonic over the total response.

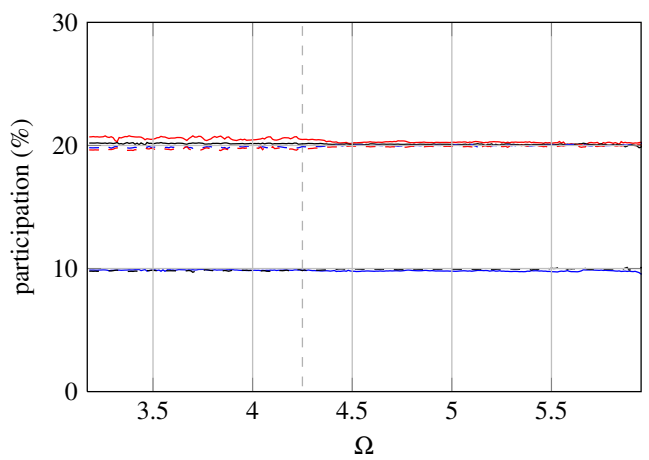

(a) $\mathrm{EO}=2$

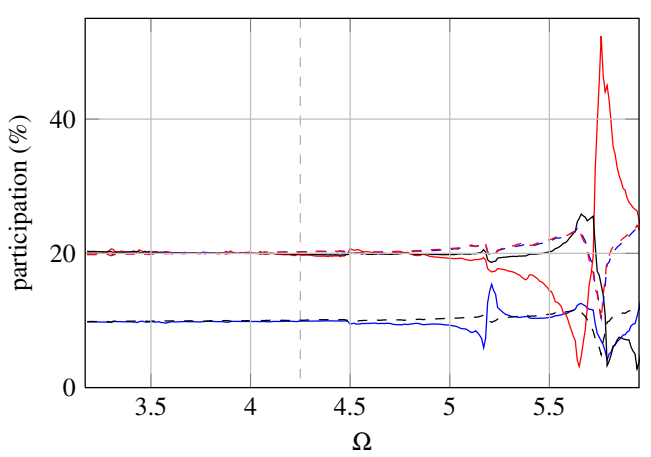

(b) $\mathrm{EO}=4$

Figure 5 - NODAL DIAMETER TRACKING FOR A 2-LOBE INTERACTION (SINGLE BLADE), $n_{d}=0(-), n_{d}=1(-), n_{d}=2(-), n_{d}=3(---), n_{d}=4(---)$ AND $n_{d}=5(---)$.

Figures. 5(a) and 5(b) respectively picture the influence of the six nodal diameters of the impeller for EOs 2 and 4 over the rotational frequency considered. It is remarkable that all the nodal diameters of the impeller are involved in the response through out the rotational frequency range. In the case of EO 2, the participation of each nodal diameter remains constant for all speeds.

For both EO 2 and 4, the participation of all the nodal diameters is practically constant for $\Omega<5$. Over this rotational frequency range, the participation of all the nodal diameters of the structure actually underlines that the vibrations are localized on the contacting blade as illustrated in Fig. 6 . To the contrary, a variation of the nodal diameters influence appears for EO 4 around $\Omega=5$ and nodal diameter 1 becomes dominant for $\Omega \simeq 5.75$. This indicates 


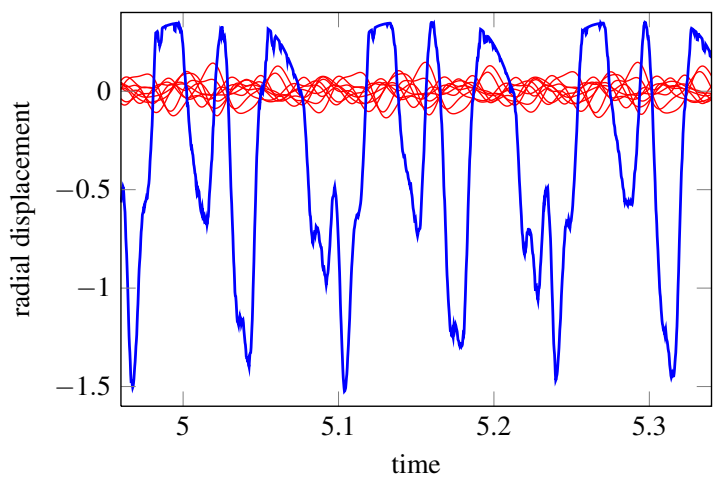

Figure 6 - LEADING EDGE RADIAL DISPLACEMENT AT $\Omega=4.25$ : CONTACTING BLADE $(-)$ AND OTHER BLADES (- - .

that even if the impeller is sollicitated on a single blade, it may respond over a first nodal diameter mode, thus hinting a non-negligible displacement of all the blades. In that case, the hypothesis of a single blade impacting the casing becomes null and void for a perfectly tuned impeller. Consequently, results for the single blade configuration are only relevant for $\Omega<5$.

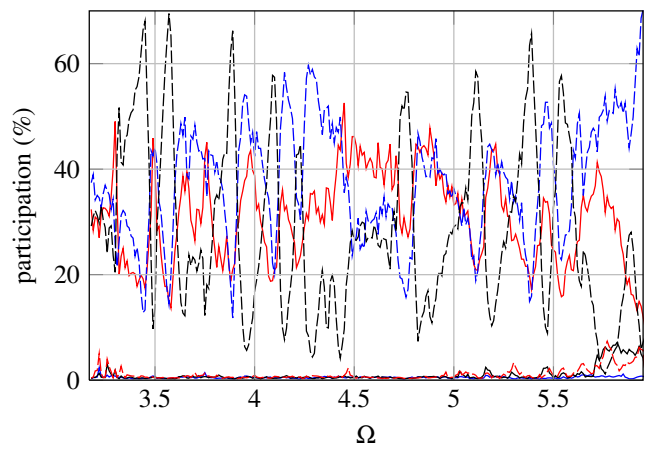

(a) $n_{d}=1$

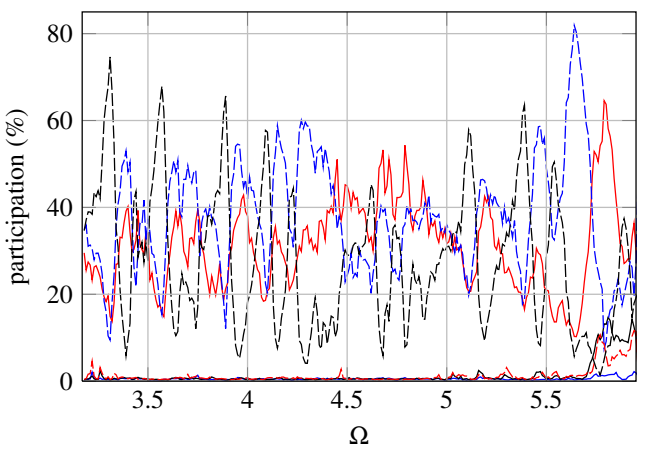

(b) $n_{d}=2$

Figure 7 - ENGINE ORDER TRACKING FOR A 2-LOBE INTERACTION (SINGLE BLADE), $\mathrm{EO}=1(-), \mathrm{EO}=2(-), \mathrm{EO}=3(-), \mathrm{EO}=4(---), \mathrm{EO}=5(---)$ AND EO $=6(---)$.

Figures 7(a) and 7(b) underline the influence of each EO when considering nodal diameters 1 and 2. Consistently with the symmetric deformation of the casing, only the even nodal diameters play a significant role in the impeller dynamics. Though it should be noticed that EO 3 becomes non negligible for high rotational frequencies $(\Omega>5.75)$ which indicates the arisal of sophisticated interaction motions.

In Fig. 3, many interaction speeds are identified for $\Omega<5$. For these speeds, results given in this section underline that there is no dominant nodal diameter and that vibrations are localized in the impacting blade. Accordingly, numerical results suggest that an interaction phenomenon involving a single blade of the impeller, similarly to what was experimentally observed in [1] for axial compressors, is plausible.

\section{Multi blade simulations}

In this section, contact is managed on all the main blades of the impeller. Similarly to the single blade case, results are first pictured in the frequency domain in order to provide the interaction map displayed in Fig. 8. 


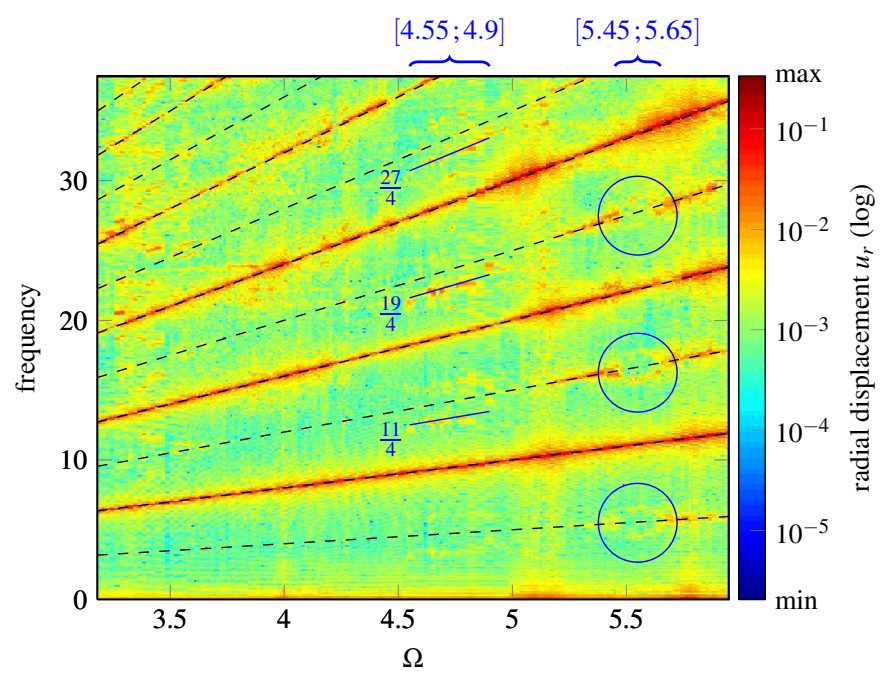

Figure 8 - INTERACTION MAP FOR 2 LOBES (MULTI BLADE CONFIGURATION).

\subsection{Frequency domain}

The colour code is identical to the one used in Fig. 3. It is noticeable that the number of interaction speeds seems reduced in comparison to what was predicted for the single blade configuration in Fig. 3. It is actually much more arduous to determine which nodal diameter of which modal family is responsible for the observed interactions. The peaks of amplitude are observed over wider rotational frequency ranges — such as $\Omega \in[5.05 ; 5.25]$ and $\Omega \in[5.2 ; 5.4]$ - and the modal density ${ }^{1}$ combined with a priori unforeseeable contact stiffening may only lead to speculations.

It is visible in Fig. 8 that a few peaks arise for non interger EOs: for instance over $\Omega \in$ $[4.55 ; 4.9]$, high amplitude of vibrations are detected along EOs $\frac{11}{4}, \frac{19}{4}$ and $\frac{27}{4}$ (solid blue lines). Also, significant amplitudes of vibration are observed along EOs 1,3 and 5 for $\Omega \in[5.05 ; 5.95]$ while significant peaks are only expected along even EOs based on linear considerations. Finally, a frequency split phenomenon is observed for $\Omega \in[5.45 ; 5.65]$ where three circles are visible around EOs 1, 3 and 5 (inside the blue circles). These observations underline the complexity of the analysis of the results in the frequency domain. In particular, linear considerations become insufficient for the prediction of interaction areas.

Consequently, results must be analyzed accounting for the symmetry of the structure and, similarly to what was made in the previous section for the single blade configuration. Harmonic tracking in space and time is advantageously used for the analysis of the results.

\subsection{Harmonic tracking}

Figures 9(a) and 9(b) picture the participation of each nodal diameter respectively considering EO 2 and 4. In agreement with the ovalized profile of the casing, even nodal diameters are dominant. In particular, nodal diameter 2 participation in the response along EO 2 is over $90 \%$ through out almost all the rotational frequency range considered. When looking at EO 4 , it is visible that nodal diameter 4 is dominant to the exception of $\Omega>5.5$ where its participation drops to less than $40 \%$. This drop matches the highest peaks of amplitude of vibration in Fig. 8 and indicates a multi-nodal diameter vibratory response of the impeller.

Similarly, figures 10(a) and 10(b) feature the participation of the six first EO for nodal diameters 2 and 4 . The participation of the second nodal diameter is essentially concentrated on EO 2. Interestingly, Fig. 10(b) exhibits an alternated participation of the fourth nodal diameter between EO 4 and 6.

Contrary to what was observed for the single blade configuration, there is an obvious relationship between time (EO) and spatial (nodal diameters) harmonics. When a peak of

${ }^{1}$ Defined here as the number of eigenfrequency lines crossing an EO at the vicinity of a peak. 


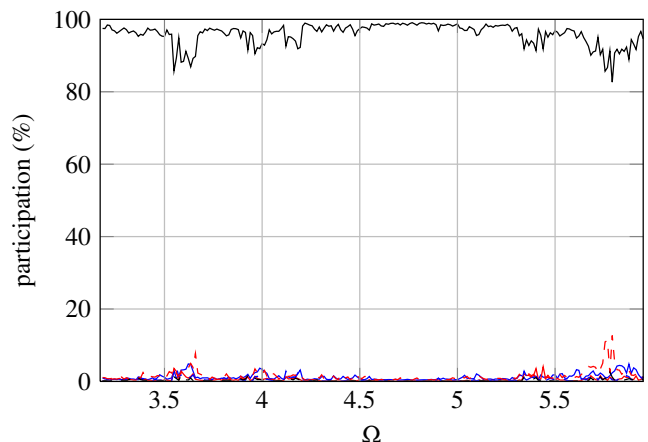

(a) $\mathrm{EO}=2$

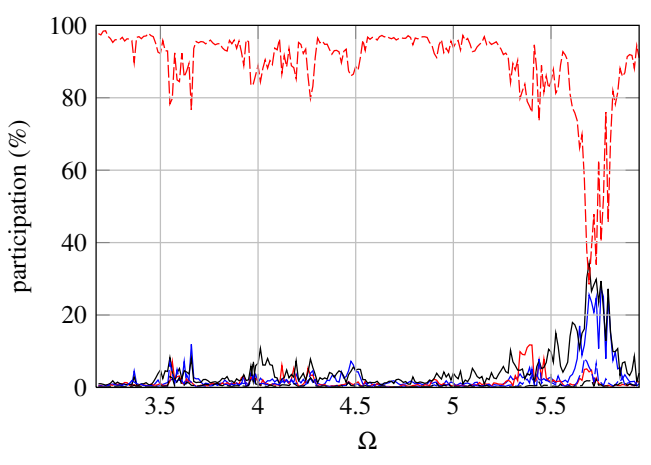

(b) $\mathrm{EO}=4$

Figure 9 - NODAL DIAMETER TRACKING FOR A 2-LOBE INTERACTION (MULTI BLADE), $n_{d}=0(-), n_{d}=1(-), n_{d}=2(-), n_{d}=3(---), n_{d}=4(---)$ and $n_{d}=5(---)$.

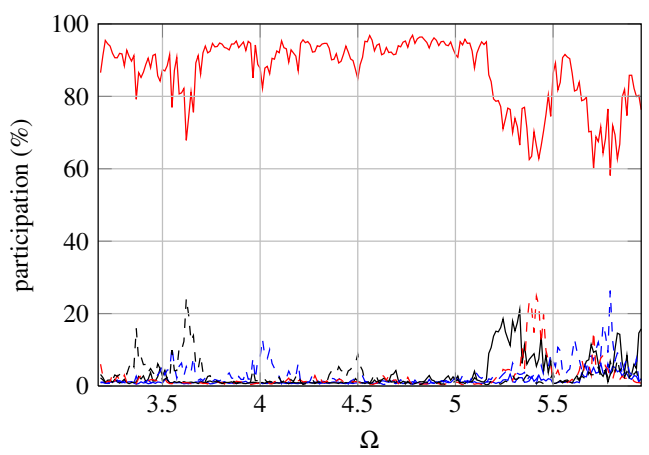

(a) $n_{d}=2$

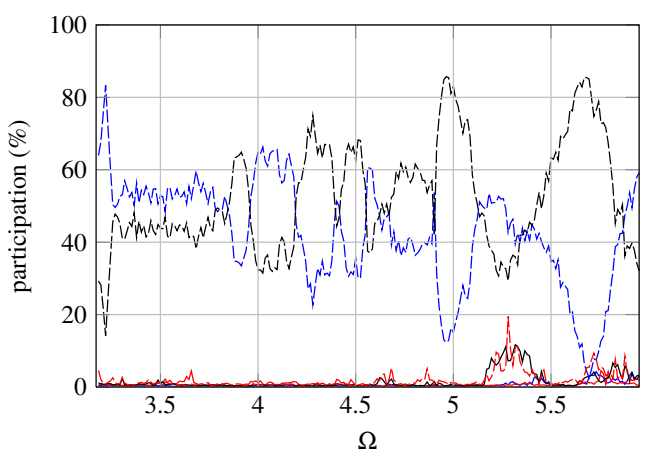

(b) $n_{d}=4$

Figure 10 - ENGINE ORDER TRACKING FOR A 2-LOBE INTERACTION (MULTI BLADE), $\mathrm{EO}=1(-), \mathrm{EO}=2(-), \mathrm{EO}=3(-), \mathrm{EO}=4(---), \mathrm{EO}=5(---)$ AND EO $=6(---)$

amplitude appears in Fig. 8 on a given EO, the associated number of nodal diameters $n_{d}$ is given by Figs. 9(a) and 9(b). The number $m$ of the modal family to which it belongs is obtained by looking at the eigenfrequency line $\left(m, n_{d}\right)$ the closest below the peak in Fig. 8.

The comparison of Figs. 5 and 7 with Figs. 9 and 10 highlights that the nature of the interaction speeds observed for $\Omega<5$ with the single blade configuration radically differs from those observed with the multi-blade configuration. In particular, the clear dominance of a single nodal diameter in Figs. 9(a) and 9(b) underlines that the observed interactions involve all the blades of the impeller as depicted in Fig. 11.

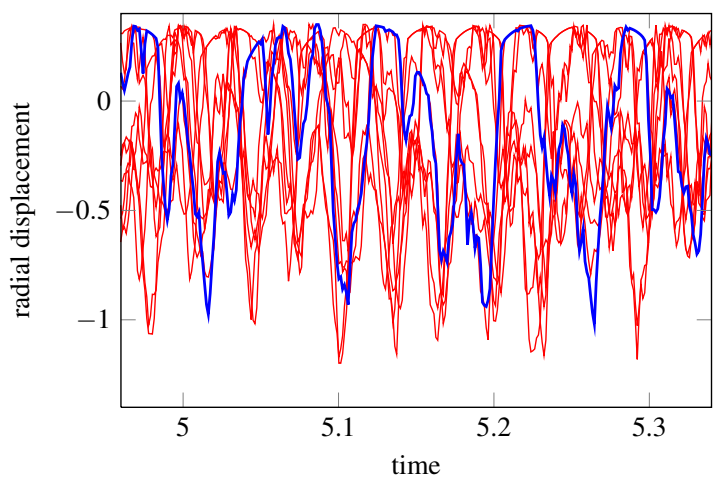

Figure 11 - LEADING EDGE RADIAL DISPLACEMENT AT $\Omega=4.25$ : BLADE 1 (-) AND OTHER BLADES (-), CONTACT IS MANAGED ON ALL THE BLADES. 


\section{Contact areas maps}

In addition to the frequency domain analysis, the numerical results may be post-processed looking at the impacted areas on the casing along its circumference. In order to do so, the casing is arbitrarily decomposed in 200 equal angular subsectors over which a numerical procedure detects if contact occurs during the fifty revolutions of the impeller. A colour code, from white (no contact) to black (one contact per revolution) indicates the number of times the first blade has impacted the casing for each rotational frequency after fifty revolutions. By doing so for three points of the blade-tip - leading edge, middle of chord and trailing edge - contact areas maps are obtained for each configuration: Figs. 12(a), 12(c) and 12(e) are relative to single blade configuration and Figs. 12(b), 12(d) and 12(f) are relative to the multi-blade contact configuration.

First of all, these maps underline the great difference in terms of vibratory behaviour between the leading edge and the rest of the blade-tip for both contact configurations. Indeed, while there are two privileged contact areas on the casing, Figs. 12(a) and 12(b) reveal that the leading edge impacts the casing four times per revolution for almost each rotational frequency. In addition, the position of the contact areas depends on the rotational frequency contrary to what may be seen in Figs. 12(c), 12(e), 12(d) and 12(f): contact areas around the middle of chord and the trailing edge are constrained by the position of the privileged contact areas $(\theta \simeq 1.71 \mathrm{rad}$ and $\theta=4.85 \mathrm{rad})$. When other contact areas are detected, such as for $\Omega \simeq 5$ and $\Omega \simeq 5.75$ in Fig. 12(f) the impeller exhibits a high vibration level in agreement with the interaction map in Fig. 8.

The comparison of Figs. 12(e) and 12(f) emphasizes quite clearly that the rotational frequency ranges over which the multi-blade contact configuration exhibits more than two contacting areas $(\Omega \in[5 ; 5.2]$, and $\Omega \in[5.65 ; 5.85]$ in Fig. $12(\mathrm{f}))$ are wider than in the case of the single blade contact configuration.

These results suggest that the interaction phenomena simulated with the multi-blade contact configuration may be more robust - in the sense that they are less sensitive to the value of the rotational frequency - than those simulated with the single blade configuration.

\section{Conclusion}

Based on an existing numerical strategy for the simulation of blade-tip/casing contact interactions, the presented work focuses on the numerical investigation of rotor/stator interactions induced by structural contacts between a centrifugal compressor and its surrounding casing within helicopter engines. Two contact configurations are investigated with an ovalized perfectly rigid casing. Results feature both time and space Fourier analysis that allow for an in-depth understanding of the vibratory behaviour of the impeller at each rotational frequency considered.

It is first underlined that numerical results corroborate the plausibility of interaction phenomena in centrifugal compressors similar to those observed numerically and experimentally in axial compressors. Such interaction phenomena involve essentially a single blade.

More complex interaction phenomena are also pointed out when contact is managed on all the blades of the impeller. In that case, the numerical robustness of the observed interactions seems higher. This study calls for more investigations including the modeling of the abradable liner that was proved to play a key role in the interaction phenomena within axial compressors.

Also, the modeling of a flexible casing would allow for the detection of modal interactions phenomena that are of great interest when designing centrifugal compressors.

\section{ACKNOWLEDGEMENT}

Thanks go to Turbomeca and Snecma for their technical and financial support. This work takes place in the framework of the MAIA mechanical research and technology program 


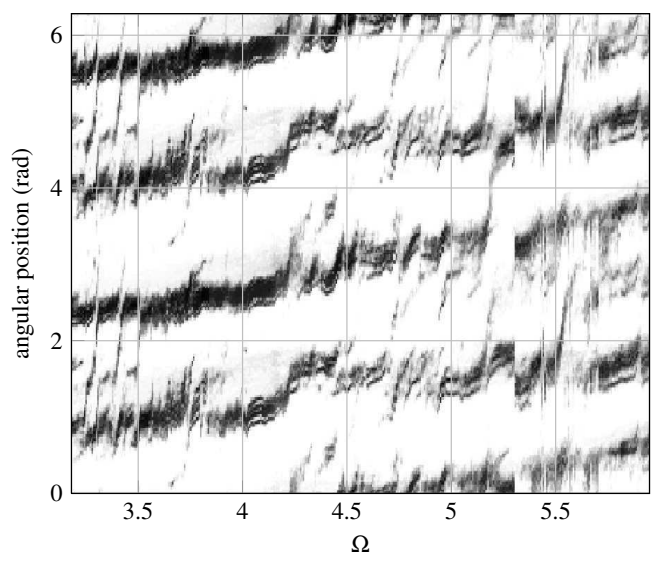

(a) Leading edge (single blade)

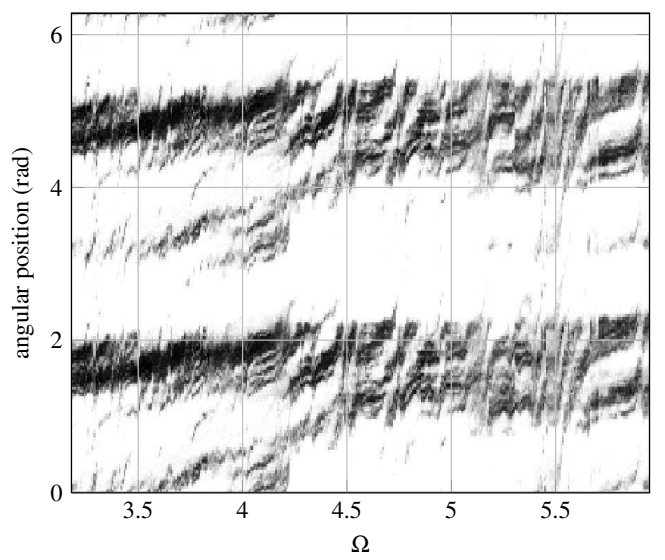

(c) Middle of chord (single blade)

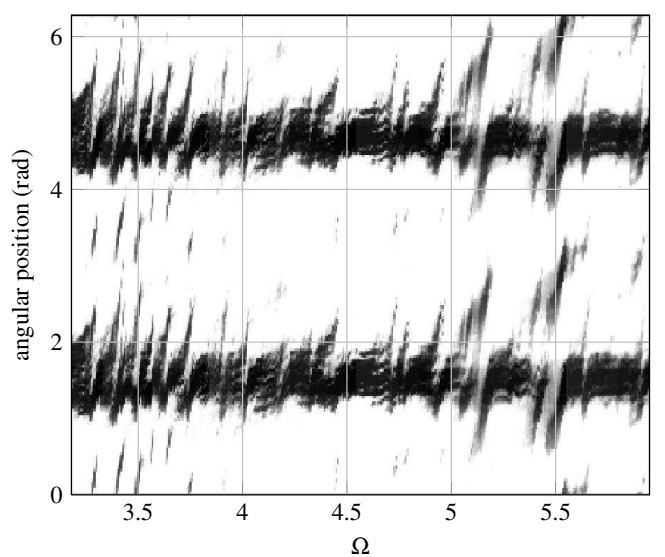

(e) Trailing edge (single blade)

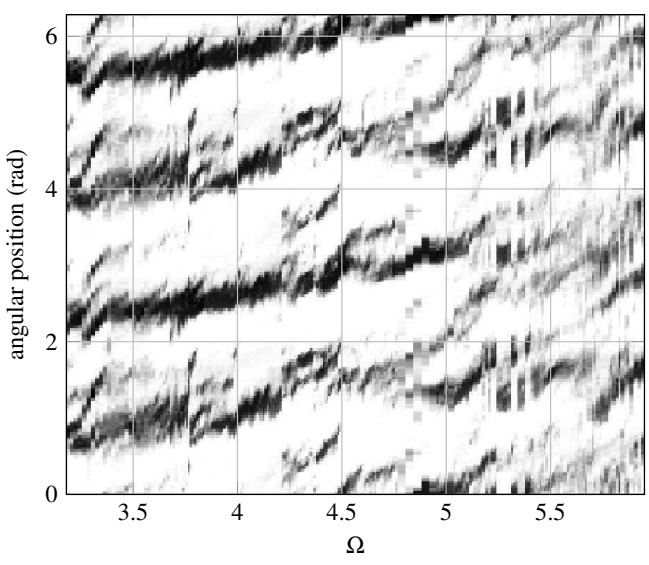

(b) Leading edge (multi blade)

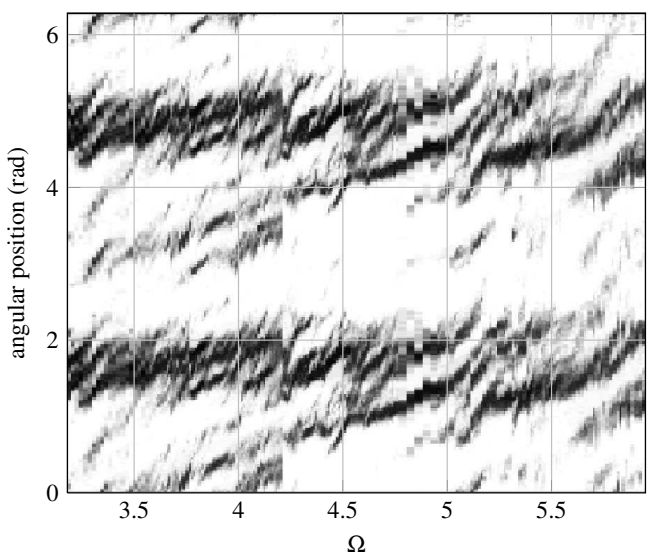

(d) Middle of chord (multi blade)

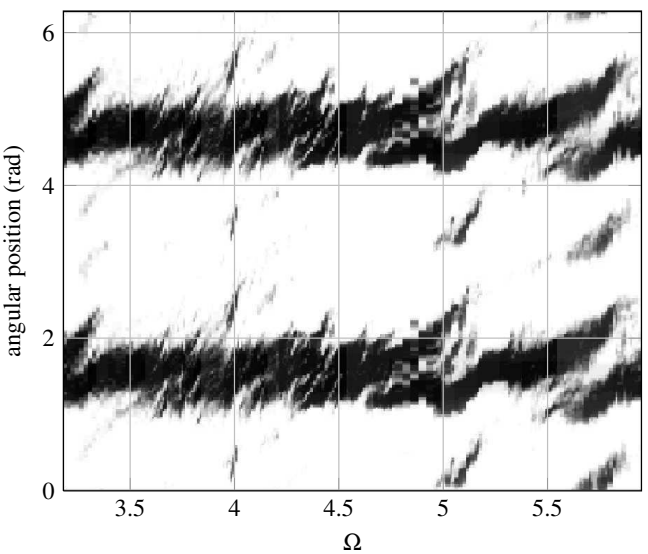

(f) Trailing edge (multi blade)

Figure 12 - CONTACT AREAS MAPS FOR THE TWO CONTACT CONFIGURATIONS OF INTEREST.

sponsored by CNRS, ONERA and SAFRAN Group.

\section{References}

[1] Millecamps, A., Brunel, J. F., Dufrénoy, P., Garcin, F., and Nucci, M., 2009. "Influence of thermal effects during blade-casing contact experiments". In Proceedings of the ASME IDETC/CIE - DETC2009-86842.

[2] Batailly, A., Legrand, M., Millecamps, A., and Garcin, F., 2012. "Numerical-experimental 
comparison in the simulation of rotor/stator interaction through blade-tip/abradable coating contact". Journal of Engineering for Gas Turbines and Power, 134(8).

[3] Legrand, M., Batailly, A., and Pierre, C., 2011. "Numerical investigation of abradable coating removal through plastic constitutive law in aircraft engine". Journal of Computational and Nonlinear Dynamics, 7.

[4] Williams, R. J., 2011. "Simulation of blade casing interaction phenomena in gas turbines resulting from heavy tip rubs using an implicit time marching method". In Proceedings of the ASME Turbo Expo 2011 - GT2011-45495.

[5] Padova, C., Barton, J., Dunn, M., and Manwaring, S., 2007. "Experimental results from controlled blade tip/shroud rubs at engine speed". Journal of Turbomachinery, 129(4), pp. $713-723$.

[6] Dadouche, A., Conlon, M. J., Dmochowski, W., Liko, B., and Bedard, J.-P., 2008. "Experimental evaluation of abradable seal performance at high temperature". In Proceedings of the ASME Turbo Expo 2008 - GT2008-51228.

[7] Padova, C., Dunn, M., Barton, J., Turner, K., Turner, A., and DiTommaso, D., 2012. "Casing treatment and blade-tip configuration effects on controlled gas turbine blade tip/shroud rubs at engine conditions". Journal of Turbomachinery, 133, pp. 011016/1 011016/12.

[8] Michimura, S., Nagamatsu, A., and Asazuma, K., 1979. "Vibration of impellers. III- an analysis of coupled vibrations between disk and blades using a reduced impedance method". JSME, Bulletin, 22(12), pp. 1293-1298.

[9] Michimura, S., Nagamatsu, A., H., Y., and Ishikawa, T., 1984. "Vibration of impellers. $\mathrm{V}$ - measurement of resonant vibratory stresses of an impeller and pressure distribution due to aerodynamic excitation”. Bulletin of the JSME, 27, pp. 534-539.

[10] Almeida, P., Gibert, C., Leblanc, X., Ousty, J.-P., and Thouverez, F., 2012. "Experimental and numerical investigations on a rotating centrifugal compressor". In Proceedings of the ASME Turbo Expo 2012 - GT2012-69760.

[11] Carpenter, N., Taylor, R., and Katona, M., 1991. "Lagrange constraints for transcient finite element surface contact”. International Journal for Numerical Methods in Engineering, 32, pp. 103-128.

[12] Legrand, M., Batailly, A., Magnain, B., Cartraud, P., and Pierre, C., 2012. "Full threedimensional investigation of structural contact interactions in turbomachines". Journal of Sound and Vibration, 331(11), pp. 2578-2601.

[13] Bladh, J. R., 2001. "Efficient predictions of the vibratory response of mistuned bladed disks by reduced order modeling". Phd thesis.

[14] Craig Jr, R., and Bampton, M., 1968. "Coupling of substructures for dynamic analysis". AIAA journal, 6(7), pp. 1313-1319.

[15] Sternchüss, A., and Balmès, E., 2006. "On the reduction of quasi-cyclic disks with variable rotation speeds". Proceedings of the International Conference on Advanced Acoustics and Vibration Engineering (ISMA), pp. 3925-3939. 